\title{
¡Que investiguen ellos! Políticas de investigación en ciencias de la salud
}

\author{
Albert J. Jovell*
}

\section{Introducción}

Se atribuye a Unamuno la paternidad de la frase uqqué investiguen ellos!n, en clara referencia a que la investigación debería ser una tarea más propia de países ricos y muy desarrollados que de países como España. A pesar de tal afirmación, en los últimos años se ha producido en España un considerable aumento de la investigación en ciencias de la salud. Los resultados de dicho aumento se han puesto de manifiesto con el incremento del número de grupos de investigación consolidados en ciencias de la salud, y con el elevado número de publicaciones científicas realizadas por científicos españoles en revistas internacionales de prestigio. A esta situación ha contribuido el desarrollo de políticas sectoriales específicas por parte de instituciones dependientes del gobierno central y de las Comunidades Autónomas (CCAA). De esta forma, las políticas promovidas por el Fondo de Investigaciones Sanitarias del Ministerio de Sanidad y Consumo (MSC) y de la CIRIT en Cataluña, por citar dos ejemplos de instituciones financiadoras de investigación sanitaria, han contribuido al crecimiento exponencial de la investigación biomédica.

Además de las políticas sectoriales de organizaciones gubernamentales, incluidas las de la Comunidad Europea, se ha fomentado la investigación sanitaria desde diferentes fundaciones y desde la industria privada. No cabe ninguna duda de que para que esta investigación fuera posible, aparte de la inversión financiera oportuna, se ha debido realizar otra inversión paralela en estructura física y capital humano. Así, y en lo referente a esta última, destacan las políticas auspiciadas por el Ministerio de Educación y Ciencia (MEC) y el MSC, así como por las CCAA y por diferentes fundaciones privadas, entre las que cabe mencionar la Fundación La Caixa y la Fundación Areces, orientadas a la formación de postgrado en el extranjero.

A todo lo antes mencionado, se ha unido la implantación y desarrollo de una cultura científica que se inicia en los centros hospitalarios universitarios y que se extiende a otras instituciones sanitarias y a los centros de atención primaria. En este sentido, la creación de la Red de Unidades de Investigación por parte del Instituto Carlos III del Ministerio de Sanidad y Consumo supuso la financiación específica de la estructura física y la contratación de capital humano, que permitió impulsar y extender la investigación sanitaria a diferentes centros del Estado español.

El objetivo de este trabajo es el de presentar las características que definen la investigación en ciencias de salud, así como plantear una propuesta de evaluación estratégica que permita valorar los elementos del proceso desde una perspectiva local. Con ello se pretende promover una reflexión crítica sobre la naturaleza y las características del proceso de investigación en España. No es objeto de este trabajo describir las diferentes políticas de investigación en ciencias de salud llevadas a cabo en el país en los últimos años, ya que éstas pueden encontrarse en las diferentes memorias de las instituciones financiadas y en publicaciones recientes (RIcoY, 1993). 


\section{Tipos de investigación en ciencias de la salud}

Al definir la investigación en ciencias de la salud, resulta conveniente distinguir los diferentes tipos y clasificaciones que se pueden realizar.

La mayor parte de las taxonomías propuestas giran en tomo a la dicotomía entre la producción de nuevo conocimiento científico y la evaluación de las aplicaciones del conocimiento ya existente. Esta dicotomía es presentada a veces como investigación básica frente a investigación aplicada y genera múltiples debates en la comunidad científica con respecto a la idoneidad de destinar fondos económicos a un tipo u otro de aproximación investigadora. Una clasificación de la investigación en ciencias de la salud que permite superar esta dicotomía ha sido propuesta por ADAY y cols. y se describe en la Tabla 1 (ADAY et al., 1993). En la misma, se dividen las diferentes categorías o tipos de investigación según el objeto de estudio.

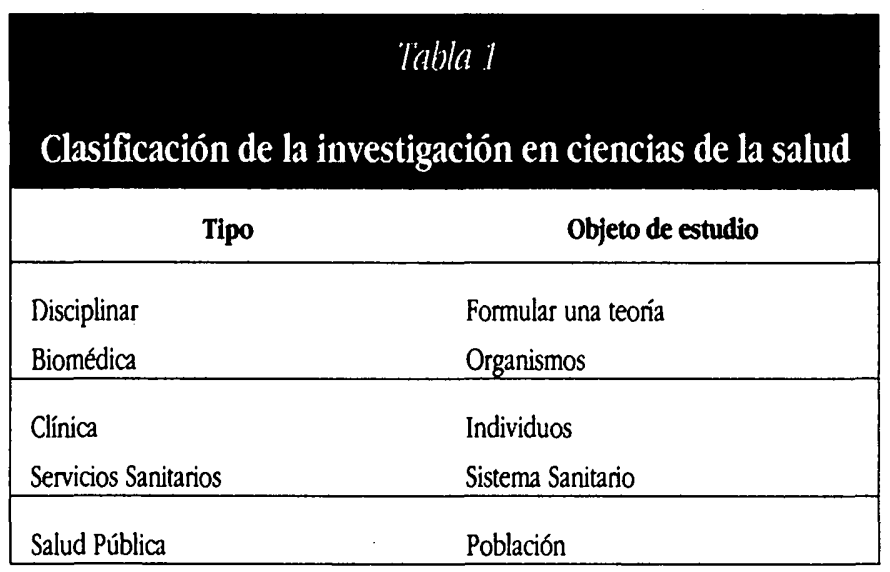

ADAY et al.; 1993.

En esta clasificación, la investigación disciplinar sería aquella orientada a determinar las bases teóricas de un fenómeno biológico o social, mientras que la investigación biomédica se orientaría a la explicación de los fenómenos biológicos o sociales, descubriendo los mecanismos de acción o la interacción entre las estructuras subyacentes. La investigación clínica estudiaría un fenómeno biológico en una población especíica de enfermos, bien sea analizando el curso natural de la enfermedad o evaluando el impacto de una intervención orientada a variar el pronóstico de la misma. La investigación en servicios sanitarios describe y analiza las características del sistema sanitario, desde la estructura y el proceso de provisión de servicios a los resultados obtenidos. La investigación en salud pública estudiaría los efectos de la enfermedad y/o de los programas destinados a su prevención en el conjunto de la población.
También se centraría en el estudio de los factores de riesgo: para enfermar.

Otro tipo de clasificaciones de la investigación en ciencias de la salud distinguen la taxonomía antes expuesta en tres grandes grupos: básica, clínica y poblacional. La investigación básica se centraría en el estudio de las bases moleculares de los procesos fisiológicos y patológicos y su lugar de acción estaría situado en el laboratorio. Este tipo de investigación analizaría fundamentalmente muestras de organismos vivos, bien sean de procedencia animal o humana, normales o patológicas. La investigación clínica analizaría el impacto de intervenciones sanitarias especíícas, por ejemplo fármacos, en la modificación del curso natural de una enfermedad. La investigación poblacional o de salud pública tendría como unidad de análisis la población, y cubriría un amplio espectro de posibilidades, que van. desde el estudio de los factores de riesgo para enfermar hasta el análisis del impacto en la comunidad.

La sistemática de clasificación de la investigación en ciencias de la salud tiene su importancia, ya que se utiliza como eje $\mathrm{d} \epsilon$ distribución de las diferentes cuotas de financiación de las politicas de investigación (CELADA, 1996). Así, las prioridades de investigación pueden orientarse hacia tipos específicos de investigación, existiendo políticas de apoyo destinadas a áreas temáticas definidas, como por ejemplo a la investigación en servicios sanitarios (GAUS y FraSER, 1996).

Otros tipos de clasificación de la investigación en ciencias de la salud se centran en áreas temáticas concretas, que a veces constituyen la base de convocatorias específicas. Así, las áreas de cáncer, cardiovascular o enfermedades neurodegenerativas han dispuesto de políticas específicas públicas o privadas de investigación temática. Un ejemplo de este tipo de convocatorias lo constituyen las organizadas anualmente por la Fundació La Marató de TV3. También, se puede realizar una clasificación en función de quién realiza la investigación o el lugar donde ésta se realiza. Así, como ejemplo de la primera aproximación podemos distinguir la diferenciación que se hace entre grupos consolidados, referido a aquellos grupos de investigación que disponen de una historia y experiencia previa acreditada en un área de estudio específica, o por consolidar, en referencia a un nuevo grupo de investigación. Un ejemplo de la segunda aproximación lo constituiría la división de la investigación según ésta se realice en centros universitarios, en instituciones sanitarias o en la industria de investigación y desarrollo (I+D).

Además de las clasificaciones mencionadas en este apartado, pueden existir otras en función de los objetivos de la investigación o de las políticas promotoras de la misma. A pesar de estas clasificaciones, hay investigadores que defienden la desaparición de las mismas, sobre todo de la frontera entre la investigación básica o molecular y la aplicada o clínica. 


\section{El proceso de investigación en ciencias de la salud}

El proceso de investigación en ciencias de la salud no tiene por qué ser muy diferente desde una perspectiva genérica al que se realiza en otros sectores. El mismo debería contemplarse como un proceso de gestión del conocimiento que se podría dividir en las cuatro fases descritas en la tabla 2.

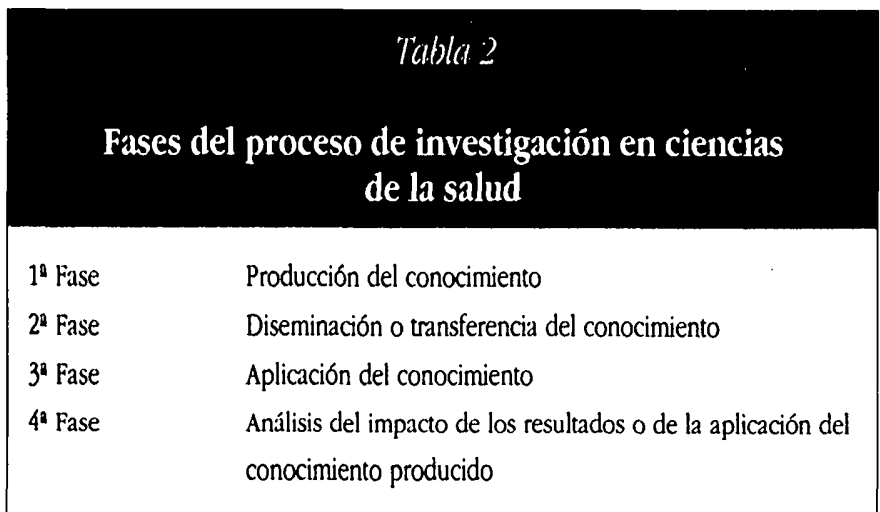

La primera fase, o de producción del conocimiento, precisa para su realización, fundamentalmente, de investigadores cualificados, de medios para realizar la investigación y de financiación adecuada. El proceso de formación de un investigador precisa de una organización universitaria adecuada, tanto en lo que respecta a los programas académicos como a su vinculación a grupos de investigación activos, a los que poder incorporar a los investigadores. En este sentido, las universidades a través de los programas de doctorado son el lugar adecuado en el que debería formar a los investigadores.

Es difícil valorar el proceso de formación de investigadores en España. El hecho de que el terreno de la investigación en ciencias de la salud sea tan amplio obliga a acotar tal valoración en función de taxonomías similares a las mencionadas en el apartado anterior de este trabajo. Así, la investigación básica, fundamentalmente en el campo de la biología celular y molecular, suele seguir un proceso de formación bien definido. Este proceso supone la integración del investigador como doctorando dentro de un programa de doctorado universitario, realizando la tesis doctoral en el seno de un grupo consolidado en una línea temática específica. Una vez finalizada la tesis, los investigadores realizan una actividad de formación postdoctoral que les permitirá inte- grarse en el futuro, si tal posibilidad existe, a una plaza de profesor universitario o en un centro de investigación de élite. El lugar de la investigación suele ser el laboratorio, y éste puede estar vinculado a una institución sanitaria o a la industria.

El proceso de formación de un investigador clínico es mucho más difícil de determinar en nuestro país. Ello es debido a la tendencia a simultanear el proceso de formación como investigador con el de médico asistencial, así como a la calidad desigual de los programas de doctorado. Es frecuente que un médico en formación en el programa MR, o ya incorporado a la red asistencial, simultanee su actividad profesional con la realización de una tesis doctoral. La valoración de las tesis doctorales como algo que hay que tener ${ }^{2}$, dentro de un mercado laboral donde existe un excedente de profesionales con méritos concurrentes que optan a una plaza de médico, y el poco interés que se muestra en la promoción de programas de doctorado de calidad podrían plantearse como hipótesis de partida para entender las tendencias actuales en el proceso formativo de un investigador clínico. A ello se une el hecho de que en los últimos años la investigación clínica se haya puesto de moda, mediante la adopción de políticas implícitas que vertebran los centros sanitarios en instituciones asistenciales, investigadores y docentes. En este sentido, las diferentes propuestas de carrera profesional incluyen la valoración de la investigación como un mérito entre los profesionales de la medicina, lo que debería llevar asociado de forma implícita una mejora de la calidad del proceso de formación de investigadores.

La formación de investigadores en salud pública sigue un proceso más diversificado. En primer lugar, porque la orientación multidisciplinar de la salud pública permite acceder a la misma desde otras profesiones diferentes a la medicina y a la enfermería, como son la economía, el medio ambiente o las ciencias sociales. En segundo lugar, porque las escuelas de salud pública existentes en el Estado español están orientadas hacia la formación de maestrías y diplomas. Estas dos circunstancias suponen, por un lado, la dificil caracterización de las disciplinas que forman la salud pública en el Estado español y, por el otro, la fragmentación de los programas de formación de doctorado, al no realizarse éstos en centros universitarios específicos de salud pública. Por otro lado, los departamentos universitarios de salud pública no parecen estar orientados a asumir la interdisciplinariedad necesaria para impartir una formación de calidad.

La descripción de los procesos de formación de investigadores realizada permite comprobar la necesidad de diseñar actividades académicas orientadas a la formación de élites investigadoras. Ello supone la elaboración y desarrollo de programas de doctorado minoritarios en tomo a grupos de investigación universitarios consolidados y con líneas abiertas de investigación adecuadamente financiadas a las que poder incorporar al investigador en formación. Además, se debería establecer la carrera de investigador, en la que se definan los elementos esenciales del proceso de formación, 
incluyendo la figura del mentor y la interacción con otros grupos de investigación en las mismas áreas temáticas, así como las estancias formativas de postgrado en centros extranjeros.

Para la realización de la carrera de investigador en ciencias de la salud resulta necesario una definición de los procesos académicos orientados a la formación de élites investigadoras. Es importante resaltar que dentro de las políticas de formación de estas élites se deberían definir los conceptos de grups:i consolidados, programa académico, carrera del investigador y persona responsable o mentor del investigador. Para ello deberían definirse programas de doctorado específicos, vinculados a grupos consolidados de investigación y a áreas financiadas acreditadas por criterios de calidad y necesidad. Obviamente, un planteamiento como el antes expuesto supone diferenciar los programas orientados a formar investigadores para seguir una carrera profesional de investigador de los destinados a enseñar cómo realizar o defender una tesis doctoral. También obliga a diferenciar la enseñanza destinada a la realización de una investigación de calidad de la orientada a completar la formación profesional de postgrado con el único propósito de aumentar la competitividad en el mercado del trabajo o para poder interpretar las investigaciones publicadas.

Un segundo aspecto relacionado con la producción de conocimiento, es la existencia de la estructura adecuada para realizar la investigación. Los elementos básicos que determinarían esta estructura aparecen citados en la Tabla 3. En los últimos años, se ha hecho un esfuerzo importante en nuestro país por facilitar la dotación estructural de los centros con el objeto de consolidar los grupos existentes y facilitar el desarrollo de nuevos grupos de investigación.

\section{Tabla 3}

\section{Elementos de la estructura investigadora}

Investigadores cualificados

Visión, misión, valores y objetivos definidos

Estructura fisica adecuada

Líneas de investigación abiertas

Financiación apropiada

Tecnología adecuada

Interacción con otros grupos de investigación

Programa de formación incorporado

Relación con el sector público $\% / o$ privado

Vinculación a los procesos de transferencia de conocimiento

Criterios de auto-evaluación y evaluación externa explícitos
Conviene distinguir la promoción de grupos orientados a la producción de conocimiento, que desde una perspectiva general entran en competencia con otros grupos de investigación a nivel mundial, de los equipos cuyo objetivo es estudiar la aplicación del conocimiento producido. En este segundo nivel, muchas veces se trabaja más a nivel local, por lo que las investigaciones realizadas en otros contextos pueden no ser vá-! lidas o generalizables al nuestro.

La segunda fase del proceso de investigación descrita en la Tabla 2 supone la diseminación del conocimiento producido a los centros de adopción del mismo. Es obvio que, para valorar esta fase, también uno debe utilizar un esquema divisorio de la investigación en ciencias de salud. Así, en investigación básica, los resultados de la misma son difundidos mediante su presentación y discusión en congresos científicos y su publicación en revistas especializadas. Es éste un conocimiento que se comparte de forma restringida entre los denominados "pares profesionales", es decir, investigadores que están trabajando en líneas temáticas similares. Ello permite la replicación de la investigación por otros grupos, si así se considera procedente, con el propósito de validar sus resultados. Por otro lado, el objetivo de este tipo de investigación es muchas veces la de transferir conocimiento, lo que facilita realizar nuevas investigaciones orientadas a la consecución de una patente y/o a alcanzar una aplicación clínica del hallazgo. Por lo tanto, no se trata de una investigación finalista de aplicación directa, sino de una investigación intermedia cuyos resultados, una vez validados y aceptados, promueven nuevas investigaciones. Así, desde el descubrimiento o caracterización de un gen hasta la utilización de ese conocimiento en el desarrollo de aplicaciones diagnósticas y/o terapéuticas se precisan múltiples investigaciones a lo largo de un horizonte temporal muy amplio.

Los resultados de la investigación clínica también se diseminan mediante su presentación en congresos específicos y su publicación en revistas científicas, al igual que en la investigación básica y en la poblacional. No obstante, el hecho de que la investigación clínica esté orientada no sólo a la producción de conocimiento sino también a testar las aplicaciones de este conocimiento en los enfermos, permite valorar la idoneidad de los procesos de transferencia de conocimiento a la práctica profesional (HAINER y DONALD, 1998). El éxito de una investigación clínica debería poder valorarse por la aplicabilidad de esos co-1 nocimientos a la mejora de la calidad de la atención sanitaria proveida a los enfermos. En este sentido, y en los últimos años, se ha promocionado activamente en ciencias de la salud los conceptos de medicina y de atención sanitaria basados en la evidencia (JOVELL y NavarRo-RUBIO, 1995a; SACKETT et al., 1997; MUIR GRAY, 1997; JOVELL, 1998; JOVELL y AYMERICH, 1999). AmbOS conceptos promueven la diseminación del conocimiento generado por las investigaciones clínicas a la práctica profesional - 
medicina basada en la evidencia - o a la planificación sanitaria -atención sanitaria basada en la evidencia-.

A pesar de estos conceptos, no se ha implantado aún en nuestro país una cultura de la evaluación de la aplicabilidad de la investigación a la resolución de problemas específicos de salud y del impacto de la misma en la mejora de la calidad del proceso de atención sanitaria. Es obvio que para poder evaluar la tercera fase del proceso de investigación o de aplicación de los resultados, se precisa esta anterior fase de diseminación, tanto en investigación clínica como en salud pública. Los elementos básicos de esta estrategia de diseminación aparecen descritos en la Tabla 4.

\begin{tabular}{|c|}
\hline $\begin{array}{l}\qquad \text { Tabla:4 } \\
\text { Elementos básicos de una estrategia de disemin }\end{array}$ \\
\hline $\begin{array}{l}\text { 1. Promover el acceso a los resultados de la investigación } \\
\text { 2. Facilitar la interpretación de las investigaciones existentes } \\
\text { 3. Diseñar estrategias educativas para los profesionales sanitarios } \\
\text { 4. Incentivar la adopción de una práctica profesional efectiva } \\
\text { 5. Auditar la práctica profesional } \\
\text { 6. Establecer una organización del trabajo que permita adoptar los resultados de } \\
\text { la investigación } \\
\text { 7. Promover la educación de los pacientes y usuarios de la sanidad } \\
\text { 8. Enfatizar el valor económico y sanitario de la investigación, así como su } \\
\text { impacto en el progreso social }\end{array}$ \\
\hline
\end{tabular}

La aplicabilidad del conocimiento producido o adquirido mediante las investigaciones clínicas y/o de salud pública depende del acceso que los profesionales puedan tener al mismo. La enorme cantidad de revistas cientificas existentes y la calidad desigual de la investigación realizada dificulta la accesibilidad a este conocimiento. A estos factores que impiden el acceso al conocimiento científico se unen otros descritos en la Tabla 5. Es por ello, que se precisan de estrategias específicas que faciliten la interpretación de la evidencia científica disponible. Así, la incorporación de las nuevas tecnologías de la comunicación deberían facilitar una accesibilidad más rápida a una información sintetizada. El desarrollo de bibliotecas virtuales y la implantación de las revistas electrónicas permiten disponer de la información en el lugar de trabajo, reduciendo los costes de búsqueda y obtención de documentos y publicaciones. Asimismo, la transferencia del conocimiento científico a la práctica profesional puede verse facilitada mediante la utilización de escalas de evaluación de la evidencia científica (JOVELL y NAVARRORuBio, 1995; Jovell et al, 1997), la elabóración de guías de práctica clínica (Jovell y NavarRo-RuBio, 1995b; Jovell et al., 1997; Jovell et al., 1998), o la formulación de recomendaciones específicas (US Preventive Services Task Force, 1996).
Tabla 5
Factores que dificultan el acceso y la utilización:dell conocimiento cientifico
1. Prevalencia de un modelo asistencial tradicional basado en la autoridad y la experiencia
2. Valores profesionales no orientados hacia la investigación
3. Ausencia de incentivos específicos
4. Organización asistencial inapropiada
5. Carencia de la educación y de las habilidades necesarias para interpretar la investigación
6. Presencia de conflictos de intereses
7. Exceso de información científica
8. Calidad desigual de la evidencia cientúfica disponible
9. Carencia de las tecnologías de información y de comunicación necesarias
10. Estrategia inapropiada de diseminación del conocimiento

No cabe ninguna duda de que para facilitar la transferencia de los resultados de las investigaciones específicas a la práctica profesional, se deben promover políticas educativas orientadas a facilitar la interpretación de dichos resultados y de entender el funcionamiento de las nuevas tecnologías. Ello debe ir asociado a políticas de organización del trabajo que favorezcan la deliberación crítica de los resultados de las investigaciones en el contexto de las sesiones clínicas y liberen tiempo de trabajo para la información, la formación y la autoevaluación. En este sentido, la diseminación del conocimiento a la práctica clínica debe vincularse a una política de recursos humanos y a un concepto de carrera profesional que incentive una medicina y una salud pública basadas en la evidencia científica. Como última fase de este proceso de investigación se debe valorar el impacto de la misma en la mejora del proceso de atención sanitaria, medido éste según indicadores de calidad asistencial, efectividad, equidad y/o eficiencia.

Finalmente, recordar, una vez más en este trabajo, que la investigación carece de sentido si sus resultados no se transfieren hacia una mejora de la práctica profesional. Es por ello que el proceso de investigación debería contemplar las cuatro fases citadas en este apartado: producción, diseminación, aplicabilidad y análisis del impacto. Cada una de esas fases debe ser objeto de políticas y estrategias específicas de gestión del conocimiento. 


\section{Evaluación de la investigación}

Un estudio de la investigación en ciencias de la salud supondría analizar las diferentes políticas y estrategias implementadas en los últimos años. Una visión globál de tales estrategias permite hipotetizar la ausencia de un plan estratégico coordinado de fomento de la investigación en ciencias de la salud. A la complejidad de definir con especificidad este tipo de investigación, lo que se ha expuesto en el segundo apartado de este trabajo, se une la fragmentación de las instituciones promotoras. Así, a la división existente entre las estructuras gubernamentales europeas, centrales y autonómicas, se unen la existencia de di- ferentes fundaciones y de la industria como instituciones que promueven investigación en este sector. Por otro lado, el sector salud presenta un gran atractivo publicitario debido a las expectativas que generan los avances del conocimiento científico en la población. Además, dentro del concepto de ciencias de la salud y de la vida, la investigación financiada comprende las áreas de Biología, Medicina, Veterinaria, Farmacología, Ciencias Sociales y Economía, entre otras.

Con el objeto de poder facilitar un mejor posicionamiento del proceso de investigación descrito en el apartado anterior, se propone en el Gráfico 1 un esquema para evaluar la investigación en ciencias de la salud. La aplicación de este tipo de esquemas permite coordinar de una forma más eficiente y efectiva la financiación de políticas de investigación en ciencias de salud.

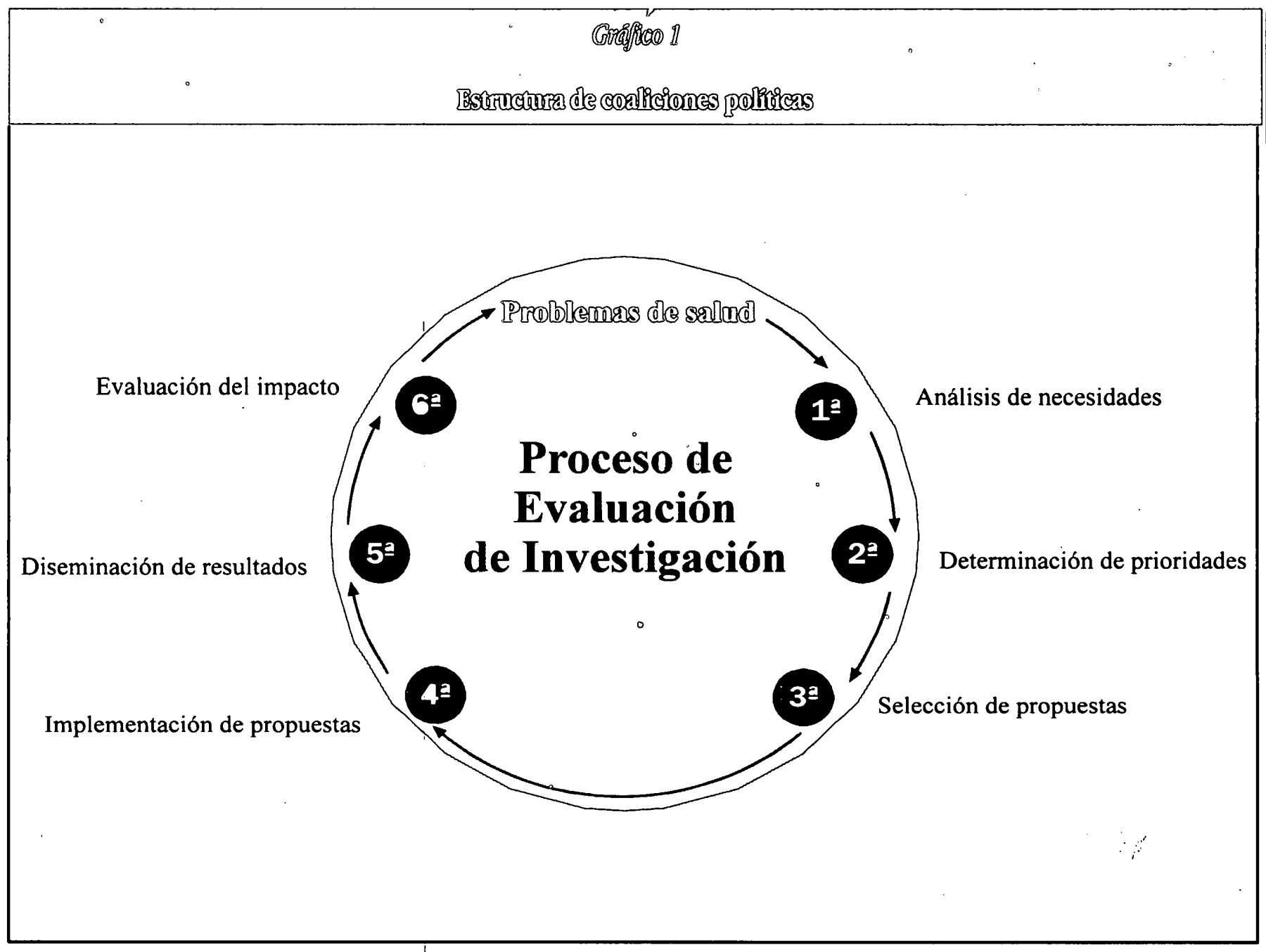

El esquema descrito parte de la presunción de que la investigación a realizar debe responder a problemas de salud específicos, de manera que los resultados de la misma pueden contribuir a la solución de los mismos. Ello supone iniciar el proceso de evaluación con un análisis de los problemas que necesitan ser investigados. Un análisis de estas características puede seguir criterios subjetivos, basados en la percepción de los expertos, u objetivos, en función de la utilización de indica- 
dores específicos mediante la aplicación de la regla utilitaria valorada según el impacto demográfico, epidemiológico o económico del problema a investigar. La metodología de análisis de necesidades en investigación no ha de ser diferente de la que se aplica en la caracterización de las necesidades de salud o de servicios sanitarios (WRiGHT, 1998). No obstante, y en el caso de la investigación sanitaria, el análisis de necesidades suele aparecer vinculado a la siguiente etapa del proceso de evaluación o de determinación de prioridades.

El proceso de determinación de prioridades en investigación en ciencias de la salud es un proceso complejo que debería ser objeto por sí mismo de investigaciones específicas. Las prioridades pueden determinarse en base a criterios muy genéricos, como por ejemplo fomentar la investigación de calidad, o muy específicos, como sería la prevención secundaria de la enfermedad coronaria. La determinación de las prioridades puede realizarse en un sentido top-down de institución promotora a centro-equipo de investigación, o bottom-up de investigador a promotor. El primer ejemplo lo constituiría en el sector público la convocatoria de un programa de investigación en servicios sanitarios mediante contratos temáticos, y en el sector privado, la contratación de los centros hospitalarios para la realización de ensayos clínicos de fármacos en investigación y desarrollo por parte de la industria farmacéutica. La determinación bottomup seguiría un esquema en el que la institución promotora determina las bases de la convocatoria, el capital disponible y, a veces, un tema o un conjunto de objetivos genéricos que permiten a los investigadores presentar sus propuestas de investigación.

La determinación de prioridades puede seguir criterios objetivos, que se corresponden a situaciones concretas definidas mediante indicadores específicos, o criterios subjetivos. La determinación subjetiva puede responder a intereses particulares de las instituciones promotoras, incluidos los objetivos políticos, a una demanda de los investigadores o a un fenómeno mediático. El proceso de determinación subjetivo puede basarse en la utilización arbitraria de indicadores objetivos, el consenso de expertos 0 en criterios establecidos a posteriori, en la fase de selección de las propuestas a financiar.

Un ejemplo de determinación de prioridades para la investigación y el desarrollo lo constituye el ejemplo del National Health Service (NHS) británico, que distingue entre criterios genéricos (Tabla 6) y específicos (Tabla 7) (BAKER y KIRK, 1996). Una revisión de los criterios de determinación de prioridades propuestos por el NHS británico permite situar como elementos principales la producción de conocimiento, la diseminación del mismo y el análisis del impacto, conceptos descritos en el apartado anterior de este trabajo. La propuesta británica promueve la aplicación de la regla utilitaria de obtención del mayor beneficio para el mayor número de personas en la determinación de las prioridades, así como la valoración a priori de la capacidad de la investigación para reducir la variabilidad y aumentar la eficiencia del proceso asistencial. Los objetivos del NHS desaconsejan la financiación de investigación minoritaria y con poca capacidad de transferencia y aplicabilidad de sus resultados, a la vez que apuestan por una investigación cuyos resultados tengan impacto específico en el proceso asistencial. De esta forma, el NHS británico tiene como objetivo una investigación eficiente (value for money) que incremente el número de proveedores participantes, que respete la libertad de los centros para realizar la labor investigadora y que permita estimar los costes del I + D y de la atención a los pacientes. En resumen, los criterios de determinación de prioridades del NHS deben responder a estas tres consideraciones:

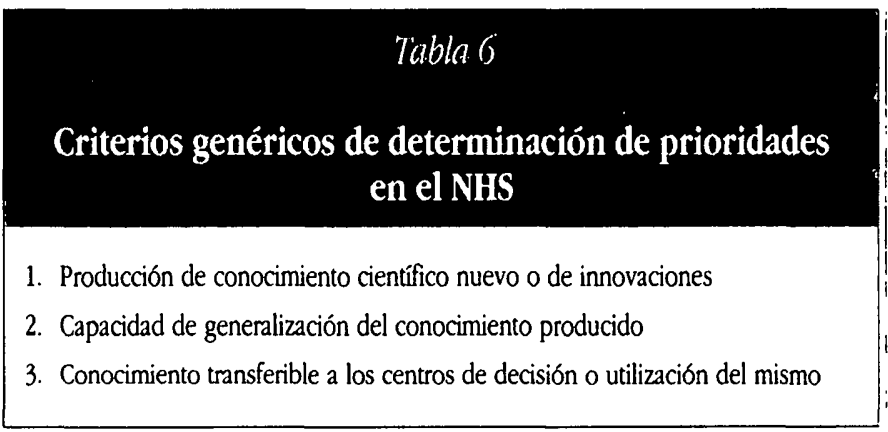

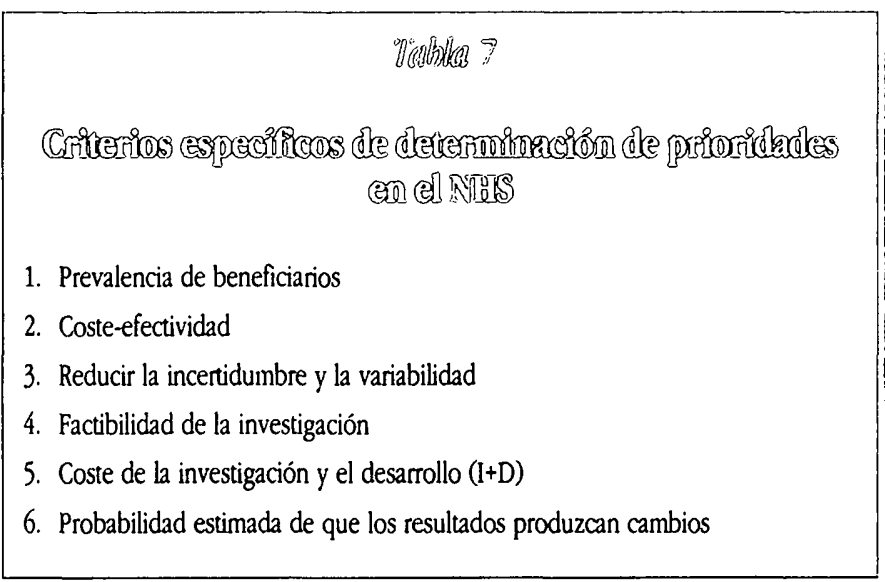

1) Necesidades identificadas mediante un proceso de análisis específico, 2) Resultados esperados responden a las necesidades de los decisores y 3) Capacidad de mejorar el proceso de atención sanitaria.

Un segundo ejemplo de determinación de prioridades lo realiza el Health Research Council holandés (RGO, 1997). Los criterios genéricos y específicos de este programa aparecen descritos en las Tablas 8 y 9. El sistema holandés determina cri- 
terios definidos de financiación según un orden jerárquico eśpecífico. A nivel del Estado español, un ejemplo de determinación de prioridades mediante la aplicación de criterios específicos se puede encontrar en las convocatorias de contratos temáticos para la investigación de servicios sanitarios de la Agencia de Evaluación de Tecnología Médica de Cataluña (Jovell et al., 1996; AATM, 1998).

\begin{tabular}{|c|}
\hline 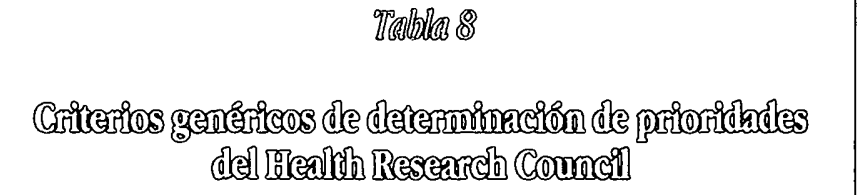 \\
\hline $\begin{array}{l}\text { 1. Contribución del conocimiento producido por la investigación básica en la } \\
\text { investigación futura } \\
\text { 2. Contribución a una mejor formación y especialización de los médicos y de los }\end{array}$ \\
\hline
\end{tabular}

\begin{tabular}{|c|}
\hline 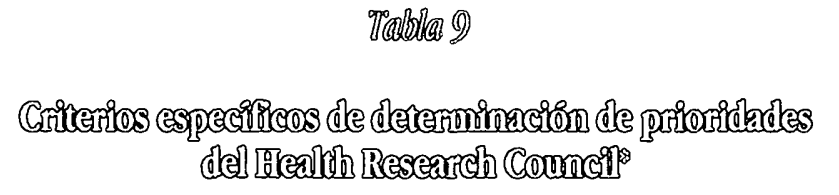 \\
\hline 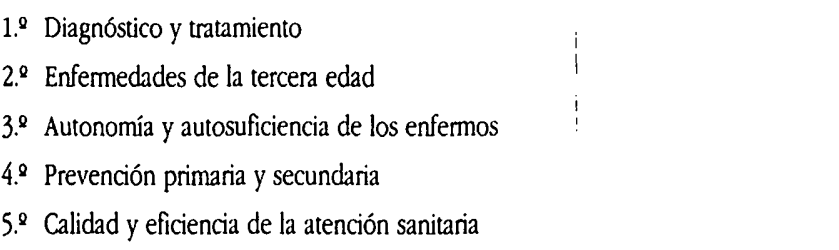 \\
\hline
\end{tabular}

- Orden jerárquico de prioridades

Las dos convocatorias realizadas por la Agencia en los años 1996 y 1998, respectivamente, establecen tres grandes grupos de prioridades: 1) Necesidad identificada en el Plan de Salud de Cataluña 1996-1998, 2) Presencia de grupos de investigación en las áreas temáticas propuestas y 3) Una lista de 15 criterios explícitos que justifican la necesidad de la investigación. Esta triple prioridad permite vincular elementos de planificación sanitaria basadas en problemas de salud de la población, con la presencia de grupos de investigación en esas áreas y el cumplimiento de criterios explícitos de priorización. Estos criterios valoran el impacto epidemiológico, económico y sanitario del problema de salud, así como la existencia de incertidumbres con respecto a la efectividad, la idoneidad, la equidad, la estandarización, la calidad y la eficien- cia del proceso asistencial. Los objetivos genéricos del programa incluyen la promoción de la investigación en servicios sanitarios en el contexto del sistema sanitario catalán, vinculando los resultados obtenidos a la evaluación y a los procesos de toma de decisiones.

La tercera fase del proceso de evaluación descrito en el Gráfico 1 es la de selección de propuestas. En esta fase se consideran tres grandes aspectos: 1) Adecuación de la propuesta a las bases de la convocatoria, 2) Adecuación de la propuesta a los objetivos de la convocatoria y 3) Competitividad de la propuesta frente a las otras alternativas presentadas. En el caso español, el proceso de selección de propuestas de investigación llevado a cabo por el MSC sigue unas normas específicas (Guallar et al., 1996). Además, el MEC tiene la Agencia Nacional de Evaluación Prospectiva, que gestiona la evaluación de múltiples convocatorias de investigación. A pesar de ello, los criterios por los que se seleccionan las propuestas de investigación en una situación de competitividad son muy difíciles de explicitar. Así, las diferentes estrategias orientadas a evitar los conflictos de interés incluyen la anonimización de las propuestas, la evaluación por dos o tres pares profesionales y la valoración en doble etapa. A pesar de estos sistemas de control, el objetivo de evitar los conflictos de intereses no tiene por qué gamantizar una evaluación simétrica de todas las propuestas, dada las dificultades en poder utilizar los mismos evaluadores aplicando los mismos criterios en la valoración de todas ellas. Por otro lado, una vez superada la evaluación administrativa, deberían tenerse claros los criterios de selección de las propuestas de estudio. En este sentido, puede existir una tendencia a favorecer un grupo de investigación consolidado o conocido en comparación a uno nuevo, y a centrarse sólo en los aspectos presupuestarios de los proyectos.

La cuarta fase o de implementación está muy relacionada con la anterior, ya que en la selección de las propuestas deben valorarse aspectos relativos a la realización de la investigación. Los criterios a tener en cuenta en esta valoración de la implementación o seguimiento de la investigación, mientras ésta se está realizando, aparecen descritos en la Tabla 10. Un criterio adicional a valorar en la selección y en la implementación de las propuestas es el hecho de que estas incluyan diferentes disciplinas, reflejo de la multidisciplinariedad de la sanidad, y se lleven a cabo en múltiples centros, lo que facilita una mayor rapidez en la obtención de resultados y en la transferencia del conocimiento producido. La fase de seguimiento o implementación está fundamentada en la confianza depositada en los investigadores con respecto a la idoneidad del proceso investigador. Sirve para detectar problemas o anticipar modificaciones con respecto a la propuesta de investigación inicial. La evaluación puede incluir auditorías específicas a los investigadores y la presentación y defensa pública de la misma frente a los pares profesionales. 


\section{Tabla 10}

Criterios de evaluación de la implementación de la investigación

1. Cumplimiento de los objetivos descritos en la propuesta

2. Aplicación adecuada de la metodología

3. Presupuesto realista

4. Adecuación y cumplimiento del proyecto propuesto

5. Resultados preliminares, si procede

Finalmente, la última fase del proceso de evaluación de la investigación sanitaria de salud es el análisis de su impacto. Esta fase de análisis está relacionada con el tipo de investigación financiada. Así, la investigación básica suele generar un conocimiento científico que es acumulable y promociona la producción de conocimiento adicional, por lo que es difícil medir sus resultados de forma tangible a corto plazo. En el caso de que la investigación produzca una innovación regulable en forma de patente, si se tiene una medida del impacto de la misma se puede valorar en términos sanitarios y/o económicos. Mientras tanto, y en ausencia de patente, la única forma de medir el impacto de dicha investigación es mediante el número y la calidad de las publicaciones científicas realizadas. Este tipo de medidas, aunque estandarizadas mediante índices bibliométricos, están sujetas a sesgos de validez y de autoría, no habiendo ninguna evidencia científica que las correlacione con una medida del beneficio generado por la inversión realizada en la investigación. De esta forma, se puede estar financiando investigación minoritaria y poco relevante que dada su originalidad puede producir un elevado número de publicaciones sin que, en ausencia de un análisis de su impacto, produzca un beneficio tangible. Además, ni los autores de las publicaciones siempre siguen criterios estándares de participación en las mismas, existiendo un espacio importante para la negociación entre los autores sobre la inclusión y el orden de los mismos, ni los editores de las revistas y los revisores de las publicaciones pueden sustraerse a percepciones subjetivas al valorar los manuscritos enviados a publicar. En este caso, la existencia de criterios formales que definen la condición de autor y la revisión de las publicaciones, no les exime de la laxitud, vulnerabilidad y discrecionalidad en su aplicación.

Algunos de los aspectos mencionados también afectan a la investigación clínica y a la de salud pública. A pesar de ello, en este tipo de investigación sí pueden determinarse criterios de impacto en base a objetivos de efectividad, idoneidad, equidad y eficiencia.
No cabe ninguna duda de que el análisis del impacto de la investigación en ciencias de la salud sólo es posible cuando se valora mediante la consecución de unos objetivos previamente definidos de forma clara, concisa, específica y cuantitativa.

\section{Conclusiones}

La clasificación de la investigación en ciencias de la salud es una tarea compleja pero necesaria, dada la multidisciplinariedad y los diferentes horizontes temporales en los que se producen los resultados. Una clasificación en tres categorías: básica, clínica y salud pública, permite situar y distinguir el proceso de investigación, aunque a veces puede ser difícil diferenciar entre los diferentes tipos. Además, resulta aún más complejo establecer distinciones entre la producción de conocimiento científico nuevo y la investigación en torno a la aplicación del conocimiento producido.

El proceso de investigación se puede dividir en cuatro fases: producción, diseminación, aplicabilidad e impacto de la investigación. La producción de investigación precisa de programas académicos estructurados de calidad orientados a la formación de investigadores. Además, se ha de formar a los profesionales a interpretar las investigaciones realizadas para que se puedan adoptar y aplicar sus resultados en la práctica profesional. La realización de investigaciones también precisa de una estructura apropiada que incluya grupos consolidados, financiación adecuada y líneas temáticas abiertas.

La diseminación de los resultados de la investigación constituye una fase esencial del proceso, ya que permite la transferencia del conocimiento científico producido a los centros de adopción y utilización del mismo. Las bibliotecas electrónicas y los centros especializados en aplicar metodologías de síntesis de la información facilitan la aplicabilidad rápida de los resultados de las investigaciones (JovelL, 1999). Finalmente, el proceso debe incluir el análisis del impacto de la investigación. Si éste fuera positivo en la consecución de los objetivos previstos, se habría justificado la realización de la misma.

La complejidad, diversidad y multidisciplinariedad de la investigación en ciencias de la salud, permite aconsejar la elaboración de planes estratégicos de investigación dentro de estrategias globales de gestión del conocimiento. En el presente trabajo se propone un esquema de evaluación de la investigación en ciencias de la salud, que pretende estimar el impacto de los resultados en responder las necesidades asociadas a una incertidumbre o problema de salud. Las tres preguntas básicas a responder serían: ¿en qué?, ¿para qué?, ¿a quién?, beneficia la investigación propuesta. 
El esquema descrito para la evaluación de la investigación incluye las fases de análisis de necesidades, determinación de prioridades, selección de propuestas, seguimiento de la investigación y análisis del impacto. La aplicación de este tipo de esquemas quizá sería útil para establecer un marco teórico para la evaluación de la investigación en ciencias de la salud. Ello per- mitiría identificar qué tipo de investigación y en qué áreas temáticas debe ser financiada en el país, sobre todo en lo referido a la investigación que si no se hace en el mismo no se hace en ninguna parte. Así, el que investiguen ellos de Unamuno puede tener muchas y legítimas excepciones.
- Director General Fundación Biblioteca Josep Laporte. Barcelona.

El autor de este artículo agradece los comentarios de María Dolores Navarto Rubio a una versión preliminar del mismo y la colaboración de Imma Guillamón en su edjción.
El presente artículo está dedicado a los compañeros de la Agencia de Evaluación de Tecnología Médica del Servicio Catalán de la Salud del Departamento de Sanidad y Seguridad Social de la Generalitat de Catalunya. como reconocimiento del trabajo realizado y el aprendizaje asociado en la preparación de dos convocatorias de investigación en servicios sanitarios.

\section{Bibliografia}

Agència d'Avaluació de Tecnologia Mèdica. Informatiu. Barcelona: AATM, 1996 y 1998.

ADAY, L.A., BeGLEY, C.H., Lawson, D.R., SLATER, C.H. Evaluating the medical care system. Effectiveness, efficiency and equity. Ann Arbor, Mi: Health Administration Press.

BAKER, M., KIRK, S. Research and development for the NHS. Abingdon, UK: Raddcliffe Medical Press, 1998.

CelaDA, A. ‘investigación clínico-básica o básico-clínica?. Med Clin (Barc) 1996;107:178-81.

GAUS, C.R., FRASER, I. Shifting paradigms and the role of research. Health Affairs $1996 ; 15: 235-42$.

HaInES, A., DONALD, A., editors. Getting research findings into practice. London: BM] Books, 1998.

Jovell, A.J., Navarro-Rubio, M.D. Evvaluación de la evidencia científica. Med Clin (Barc) 1995a;105:740-3.

Jovell, A.J., Navarro-Rubio, M.D. •Guías de práctica clínica. FMC 1995b;2:60-4.

Jovel, A.J., Granados, A., Terra, N. •Developing funding research priorities to meet policy-making needs: the experience of Catalonia. First Intermational Conference on Priorities in Health Care. Stockholm, Sweden, Octubre 1996.

Jovell, A.J., Navarro-Rubio, M.D., Aymerich, M., Serra-Prat, M. eMetodología de diseño y elaboración de guías de práctica clínica. Atención Primaria 1997; 20:259-66.
Jovell, A.J. .Medicina basada en la evidencia cienúfica. El Médico, 6 de febrero de 1998.

Jovell, A.J., Aymerich, M., GarCiA-Altés, A., SerRa-Prat, M. Guia de práctica clínica del tratamiento medicado de la infección por Helicobacter pylori asociado a úlcera duodenal en la atención primaria. Barcelona: Agència d'Avaluació de Tecnologia Mèdica, Servei Català de la Salut, Departament de Sanitat i Seguretat Social, Generalitat de Catalunya. Septiembre, 1998. [BR98002].

JOVEIL, A.J., AYMERICH, M., editores. La toma de decisiones en Sanidad: El papel de la evidencia científica. Monografía de la Academia de Ciencias Médicas de Cataluña y Baleares (en prensa).

JovelL, A.J. •Bibliotecas en ciencias de la Salud: El futuro es digital. Revista Quark (en prensa).

MuIR GraY, J.A. Atención sanitaria basada en la evidencia. Madrid: Churchill Livingstone. España, 1997.

Raad voor Gerondheidsonderroek (RGO). Health Research and Government Policy: Long-term perspective. RGO, 1997.

RicoY, J.R. .La financiación de la investigación médica. Med Clin (Barc) 1993; 100 (Supl 1):6-8.

SACKETT, D.L., RICHARDSON, W.S., ROSENBERG, W., HAYNES, S.B. Medicina basada en la evidencia. Madrid: Churchill Livingston España, 1997.

US Preventive Services Task Force. Guide to clinical preventive services (2nd edition). Alexandria, VA: Intemational Medical Publishing, 1996.

WRIGHT, J., editor. Health needs assessment in practice. London: BMJ books, 1998. 